\title{
The Accuracy of Alignment Determined by Patient- Specific Instrumentation System in Total Knee Arthroplasty
}

\author{
Kwangkyoun Kim, $\mathrm{MD}^{1}$, Jungsung Kim, $\mathrm{PhD}^{2}$, Dokyoung Lee, $\mathrm{MD}^{1}$, Sohui $\mathrm{Lim}^{2}$, and Jiyoon Eom ${ }^{2}$ \\ Departments of ${ }^{1}$ Orthopedic Surgery and ${ }^{2}$ Medical Engineering, Konyang University College of Medicine, Daejeon, Korea
}

Purpose: The aim of this study is to assess the accuracy of alignment determined by patient-specific instrumentation system in total knee arthroplasty (TKA).

Materials and Methods: Twenty-seven TKAs using patient-specific instrument were reviewed. The intraoperative pin location determined by the patient-specific guide was recorded using imageless navigation software. Data recorded included tibial coronal alignment and posterior slope, femoral coronal alignment and sagittal alignment, and transepicondylar axis. A discrepancy within $\pm 3^{\circ}$ in each plane was considered an acceptable result.

Results: On the tibia, an acceptable alignment was obtained in 24 (88.1\%) in the coronal plane and 21 (77.8\%) in the sagittal plane. On the femur, a satisfactory alignment was obtained in $25(92.6 \%)$ in the coronal plane and $24(88.1 \%)$ in the sagittal plane. Based on the transepicondylar axis, a satisfactory alignment was obtained in $23(85.1 \%)$.

Conclusions: Satisfactory alignment was obtained in more than $85 \%$ of each plane of the femur and in the coronal plane of the tibia and relative to the transepicondylar axis. Sufficeint experience and precise preoperative planning are required to improve the accuracy of sagittal alignment of the tibia.

Keywords: Knee, Arthroplasty, Computer assisted, Patient specific instrument

\section{Introduction}

Restoration of the mechanical axis is one of the most important factors influencing the longevity of implants in total knee arthroplasty (TKA). The most commonly used instruments in TKA are the extramedullary alignment guide for tibial resection and the intramedullary alignment guide for distal femoral resection. They have been used for decades, are relatively easy to use, and

Received June 8, 2018; Revised September 7, 2018;

Accepted September 27, 2018

Correspondence to: Kwangkyoun Kim, MD

Department of Orthopaedic Surgery, Konyang University College of

Medicine, 158 Gwanjeodong-ro, Seo-gu, Daejeon 35365, Korea

Tel: +82-42-600-9862, Fax: +82-42-545-2373

E-mail: kimajouos@gmail.com

This is an Open Access article distributed under the terms of the Creative Commons Attribution Non-Commercial License (http://creativecommons.org/licenses/by-nc/4.0/) which permits unrestricted non-commercial use, distribution, and reproduction in any medium, provided the original work is properly cited. are familiar to most knee surgeons. However, they have been associated with limited accuracy for various reasons such as variation in the femoral neck-shaft angle in individuals, mismatch between the width of the femoral canal and the intramedullary rod, inaccurate placement of the jigs, and wrong bone cuts.

Patient-specific instruments (PSIs) were recently introduced to increase the accuracy of the surgical technique and avoid issues related to the complexity of the navigation system, such as procedural costs, surgical time, and learning curve. However, postoperative alignment following TKA with use of PSIs showed conflicting results $^{1-4)}$. This study investigated the accuracy of alignment associated with a PSI manufactured at the author's institution for TKA.

\section{Materials and Methods}

In the present study, 27 patients treated surgically at the authors' institution for TKA using the PSI between June 2017 and December 2017 were enrolled and analyzed. The inclusion criteria 
were the diagnosis of primary osteoarthritis, no history of open knee procedures, an extension deficit less than $20^{\circ}$, and coronal deformity less than $15^{\circ}$. The PSI for TKA was manufactured by a 3D printer (Objet Connex 500, Rehovot, Israel) at the authors' institution and registered by the Korean Ministry of Food and Drug Safety. All surgical plans were specifically made, modified, and approved by a single surgeon (KK) before surgery. Our institution's Ethics Committee approved the study, and informed consent was obtained from all patients (KYUH 2017-10-008).

\section{Reconstruction of Three-Dimensional (3D) Model}

Using an 80-channel computed tomography (CT) (Aquilion PRIME, Canon, Tokyo, Japan), 1-mm-thick axial CT images of the hip, knee, and ankle were obtained preoperatively in all patients. Computer software (Mimics ver. 19; Materialise, Leuven, Belgium) was used to generate a 3D model of the patient's anatomy via thresholding and 3D CT reconstruction.

\section{Preoperative Determination of Axes for Alignment}

The mechanical axis of the femur was determined by connecting the center of the femoral head and the femoral knee center. In the tibia, the mechanical axis was determined by connecting the tibial knee center and the ankle center (Fig. 1A-C). The surgical transepicondylar axis was used for femoral component rotation (Fig. 1D).

\section{Proposed Bony Resection and Determination of Pin Location}

Proposed bony resections to obtain the desired coronal and rotational alignments were templated. Using computer software (SolidWorks, Chicago, IL, USA), a bony resection was made perpendicular to the femoral mechanical axis in the coronal and sagittal planes. In the tibia, coronal bony resection was made perpendicular to the mechanical axis of the tibia, and the posterior tibial slope was set to $3^{\circ}$ in the sagittal plane. In addition, the rotation of the femoral component was set parallel to the surgical transepicondylar axis ${ }^{5,6)}$. The PSI was used as a pin guide to position the manufacturer's conventional cutting blocks, and pin locations were determined according to pre-planned alignment and bony resection thickness (Fig. 2).

\section{Measurement of the Accuracy of Pin Location}

Accuracy of pin location was determined intraoperatively to record alignments before bone sawing. Knee navigation software (Stryker Co., Allendale, NJ, USA) was used to assess the pin location with respect to the corresponding preoperative plan. Registration of anatomical landmarks for alignment in the navigation-assisted TKA was the same as preoperative planning for TKA with the PSI. Differences were that the hip center was registered with circumduction of the hip with changing radii and both malleoli and both epicondyles were covered with soft tissue in navigation-assisted TKA. The accuracy was measured by plac-

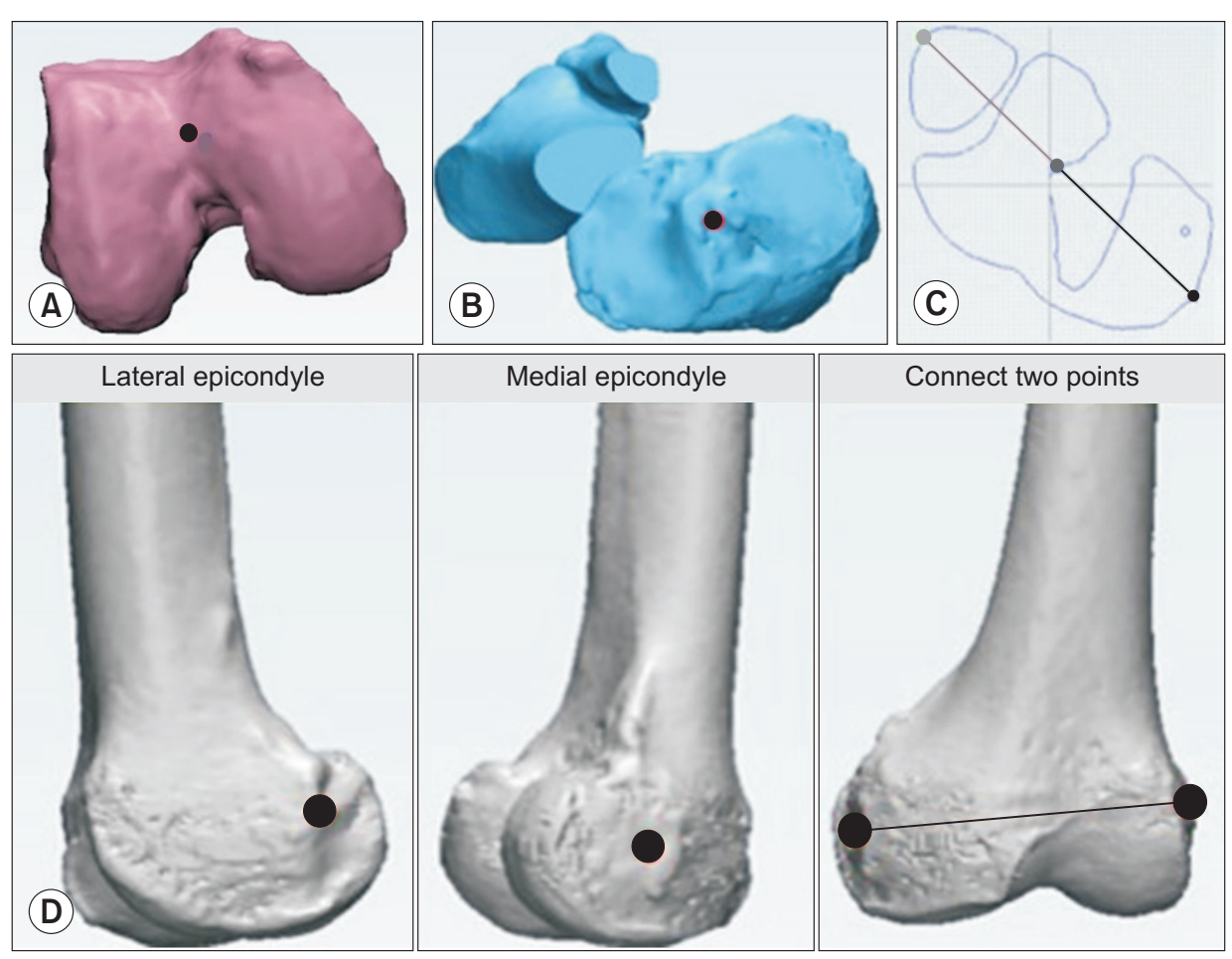

Fig. 1. Preoperative surgical plan for the use of the patient-specific instrument based on preoperative computed tomography. (A) The femoral knee center was marked at the center of the trochlear sulcus anterior toward the distal end of the femoral shaft. (B) The Tibial knee center was marked in the middle of the interspinous sulcus. (C) The ankle center was located midpoint between the most protruding points on the medial malleolus and the lateral malleolus. (D) The surgical transepicondylar axis passes through the medial epicodyle (sulcus) to the lateral epicondyle (eminence). 


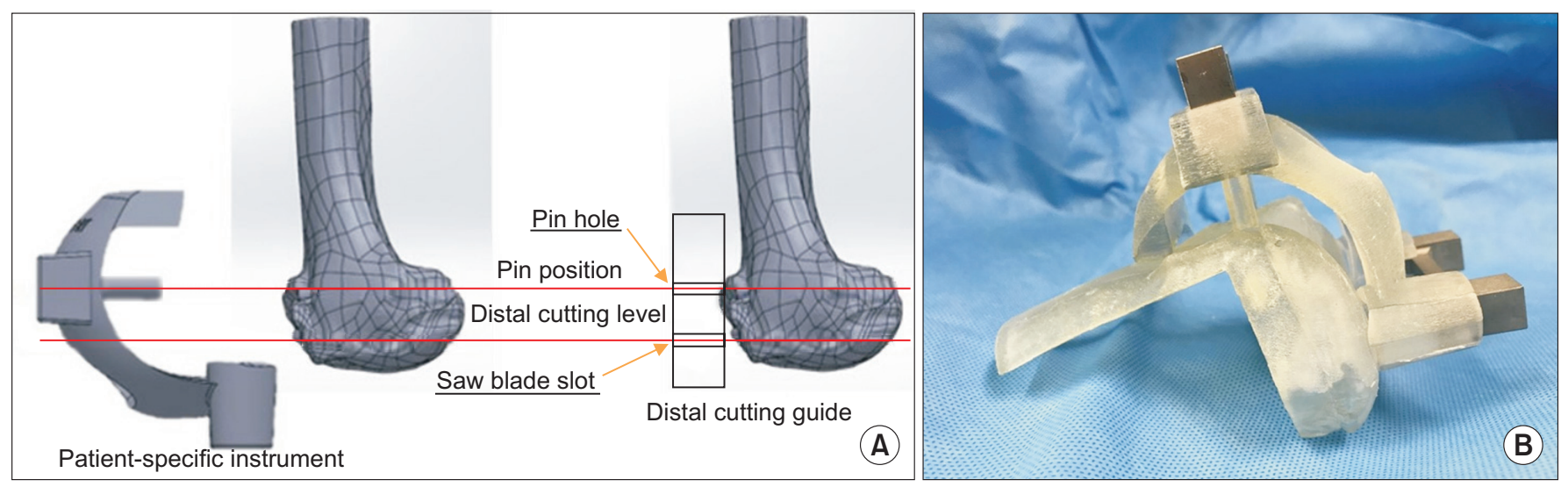

Fig. 2. (A) The patient-specific instrument (PSI) was manufactured by considering the distance between the pin hole and the saw slot location of the cutting block of the implant of total knee arthroplasty. (B) PSI for the femur manufactured at the authors' institution.
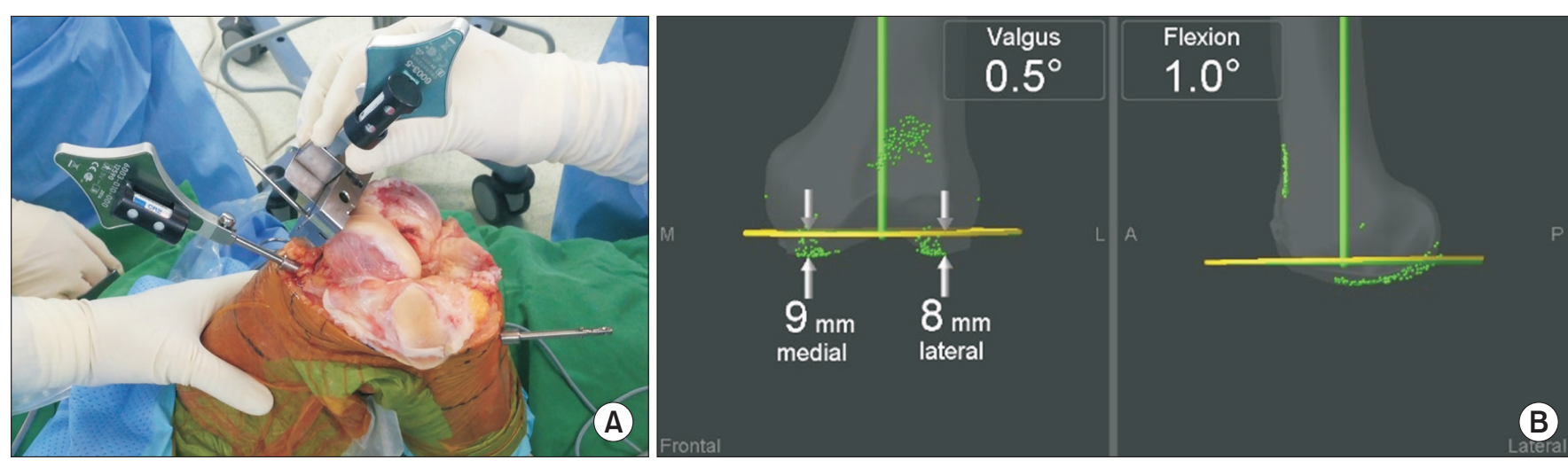

Fig. 3. (A) Evaluation with use of the navigation system. (B) Navigation system showing data in both planes.

ing the navigation instrument into the slot of the conventional cutting block, and any deviation from the planned resection was recorded on the monitor (Fig. 3). The tibial coronal alignment and posterior slope, femoral coronal and sagittal alignments, and surgical transepicondylar axis were determined. A discrepancy within $\pm 3^{\circ}$ in each plane was considered acceptable.

\section{Results}

In the tibial coronal plane, the mean deviation from the preplanned alignment was $1.3^{\circ} \pm 1.9^{\circ}$ (range, $-4^{\circ}$ to $5^{\circ}$ ). A positive value indicates a deviation in varus alignment from the preplanned alignment and a negative value suggests valgus alignment. In the tibial sagittal plane, the mean deviation from $3^{\circ}$ of posterior slope was $1.6^{\circ} \pm 2.0^{\circ}$ (range, $-2^{\circ}$ to $5^{\circ}$ ). Positive values indicate a more posterior slope from the pre-planned alignment and negative values, a more anterior slope. In the femoral coronal plane, the mean deviation from the pre-planned alignment was $0.8^{\circ} \pm 1.9^{\circ}$ (range, $-4^{\circ}$ to $4^{\circ}$ ). Positive values indicate a deviation in varus alignment from the pre-planned alignment and negative values suggest a valgus alignment. In the femoral sagittal plane, the mean deviation from the pre-planned alignment was $0.39^{\circ} \pm 2.1^{\circ}$ (range, $-3^{\circ}$ to $4^{\circ}$ ). Positive values indicate a flexion from the pre-planned alignment and negative values indicate an extension. Referring to the surgical transepicondylar axis, the mean deviation from the pre-planned alignment was $0.2 \pm 2.1$ (range, $-4^{\circ}$ to $4^{\circ}$ ). Positive values indicate a more external rotation from the pre-planned alignment and negative values suggest an internal rotation. In the tibia, an acceptable alignment was obtained in 24 patients (88.1\%) in the coronal plane and in $21 \mathrm{pa}-$ tients (77.8\%) in the sagittal plane (Fig. $4 \mathrm{~A}$ and B). In the femur, a satisfactory alignment was obtained in 25 patients (92.6\%) in the coronal plane and in 24 patients (88.1\%) in the sagittal plane (Fig. 4C and D). Based on the surgical transepicondylar axis, a satisfactory alignment was obtained in 23 patients (85.1\%) (Fig. 4E). 
A Tibial positioning in the coronal plane

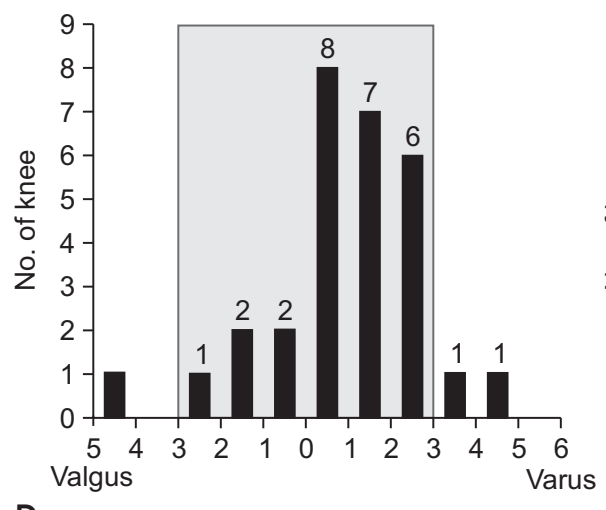

D

Femoral positioning in the sagittal plane

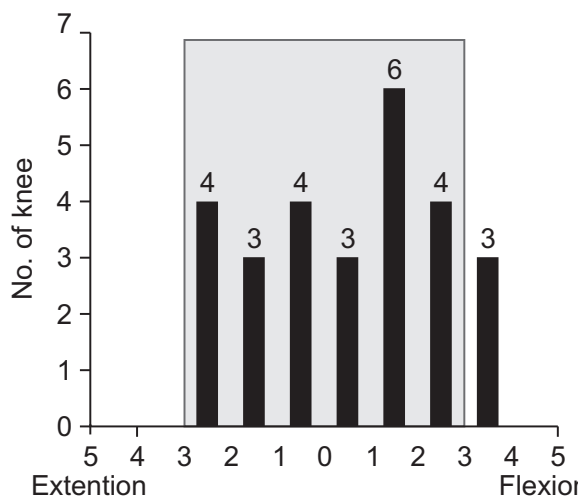

B

Tibial positioning in the sagittal plane (posterior slope)

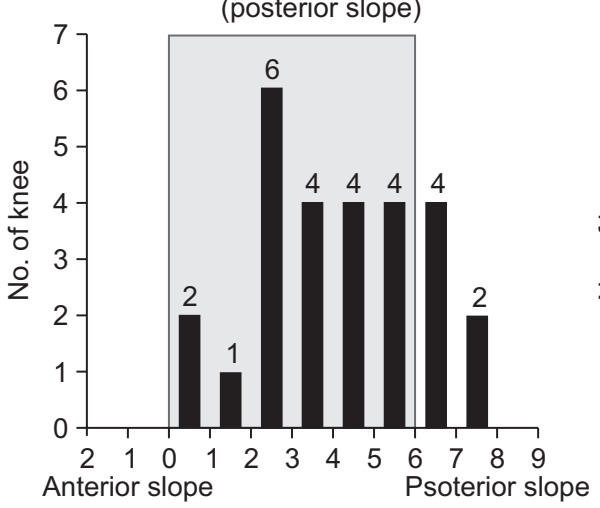

E

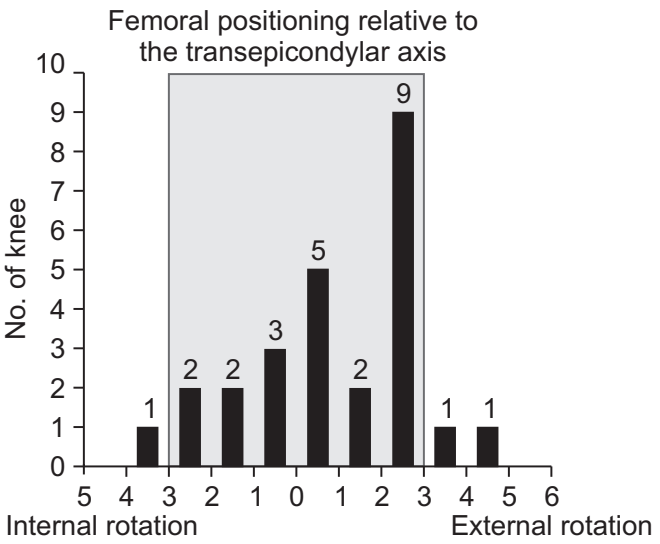

C

Femoral positioning in the coronal plane

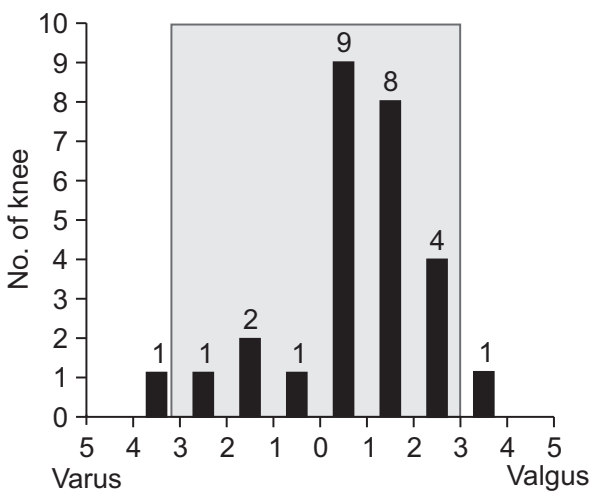

Fig. 4. (A) Alignment in the tibial coronal plane. (B) Alignment in the tibial sagittal plane. (C) Alignment in the femoral coronal plane. (D) Alignment in the femoral sagittal plane. (E) Alignment relative to the transepicondylar axis.

\section{Discussion}

PSIs were manufactured with the objective of replacing traditional instruments that need to be sterilized in the operating theater rather than simply following the patient-matched guides and the corresponding implants. However, they have demonstrated limited accuracy ${ }^{5-12)}$, and postoperative results can be affected by the methods of measurement and accuracy of preoperative planning.

Navigation-assisted TKA may improve accuracy in the coronal plane, but it is not as effective in terms of the accuracy of rotation or tibial slope ${ }^{7-9)}$. Although PSIs were reported to improve accuracy in multiple planes owing to 3D planning with either MRI or CT, few studies reported the comparative accuracy of PSIs and other instruments in the sagittal plane ${ }^{10-13)}$. In this study, the accuracy was lower in the tibia than in the femur and in the sagittal plane than in the coronal plane. The accuracy of the tibial sagittal plane was lowest, which was $77.8 \%$ similar to navigation-assisted $\mathrm{TKA}^{7-9)}$.
Three possible causes gave rise to the inferior accuracy of the coronal alignment of the tibial component than the femoral component, especially the sagittal tibial alignment. Accuracy and reproducibility of determination of the anatomical location may be one of the factors. The determination of the hip center is known to be precise ${ }^{14-16)}$. It may be more accurate to functionally determine the center of a hip joint than the ankle joint, marking the most prominent points of both malleoli. In addition, in this study, the medial parapatellar approach was used to place the PSI. In this case, the PSI was located medial to the midline because more bone contact was better for secure placement. It could have affected the accuracy of the posterior slope because of the obliquity of the cut surface. The third possible cause is related to the difference in accuracy and reproducibility of computer-assisted surgery (CAS) and PSI in the marked ankle covered with soft tissue and primarily found by direct palpation. Therefore, landmarking during navigation of the tibia cannot exactly reproduce the preoperative landmarking on the $3 \mathrm{D}$ model. Nevertheless, there are two reasons to determine the accuracy of pin location 
with knee navigation software intraoperatively. First, the assessment of accuracy using postoperative X-ray or CT image is affected by intraoperative sawing error, toggling, cut adjustment, and final impaction. Second, the change of PSI to a navigationassisted TKA may be necessary in unacceptable outlier cases.

Our results showed an accuracy of $85.1 \%$, which is comparable to corresponding values determined using conventional instrumentation $^{17-19)}$. Theoretically, based on preoperative 3D imaging, the PSI should enhance rotational component alignment, even when compared with CAS techniques. Accurate rotational alignment using CAS techniques has been considered difficult to obtain as the anatomic landmarks entered into the system intraoperatively rarely exhibit high accuracy to effectively assess the component rotation ${ }^{17)}$. In our study, we used the surgical transepicondylar axis as the reference for femoral rotational alignment. In our previous study using 3D CT, the surgical transepicondylar axis was more reliable and reproducible than the anatomical transepicondylar axis because the medial epicondyle appears as a horse's hoof with a marginal elevation measuring 10 $\mathrm{mm}$ in diameter ${ }^{6}$.

In this study, CT was used for 3D model reconstruction. MRI provides an improved image of the cartilage surfaces than $\mathrm{CT}^{19,20)}$. Deviation from the real joint surface based on the cartilage surface estimation using CT-based PSI can result in an inadequate fit between the PSI and bone and differences in bone cutting thickness during intraoperative and preplanning stages. Pfitzner et al. ${ }^{10)}$ reported that MRI-based PSI was more accurate than CTbased patient-specific instrumentation regarding the coronal mechanical limb axis, although differences were only subtle and of questionable clinical relevance. Ensini et al. ${ }^{13)}$ compared $25 \mathrm{MRI}$ based PSI TKAs with 25 CT-based PSI TKAs: they found no difference in the coronal alignment.

Several limitations of the present study should be noted. First, the patient population was small and there was no comparison with CAS or conventional instrumentation. Second, the enrolled patients had less severe deformity; therefore, the findings of the study may not be applicable to all TKA patients. Third, the use of imageless navigation system could have caused inaccuracy.

\section{Conclusions}

Satisfactory alignment was obtained in more than $85 \%$ of each plane of the femur and in the coronal plane of the tibia and relative to the transepicondylar axis. Sufficient experience and precise preoperative planning are required to improve the accuracy of the sagittal alignment of the tibia.

\section{Conflict of Interest}

No potential conflict of interest relevant to this article was reported.

\section{References}

1. Howell SM, Kuznik K, Hull ML, Siston RA. Results of an initial experience with custom-fit positioning total knee arthroplasty in a series of 48 patients. Orthopedics. 2008;31:85763.

2. Ng VY, DeClaire JH, Berend KR, Gulick BC, Lombardi AV Jr. Improved accuracy of alignment with patient-specific positioning guides compared with manual instrumentation in TKA. Clin Orthop Relat Res. 2012;470:99-107.

3. Steimle JA, Groover MT, Webb BA, Ceccarelli BJ. Acute perioperative comparison of patient-specific instrumentation versus conventional instrumentation utilization during bilateral total knee arthroplasty. Surg Res Pract. 2018;2018: 9326459.

4. Stolarczyk A, Nagraba L, Mitek T, Stolarczyk M, Deszczyński JM, Jakucinski M. Does patient-specific instrumentation improve femoral and tibial component alignment in total knee arthroplasty? A prospective randomized study. Adv Exp Med Biol. 2018;1096:11-7.

5. Berger RA, Rubash HE, Seel MJ, Thompson WH, Crossett LS. Determining the rotational alignment of the femoral component in total knee arthroplasty using the epicondylar axis. Clin Orthop Relat Res. 1993;(286):40-7.

6. Kim KK, Lee WS, Heo YM, Yi JW, Lee JB, Chae DS. Distal femoral rotational alignment based on mechanical axis of the femur: a 3-dimensional computed tomographic scan in vivo assessment. J Korean Orthop Assoc. 2011;46:484-91.

7. Cheng T, Zhao S, Peng X, Zhang X. Does computer-assisted surgery improve postoperative leg alignment and implant positioning following total knee arthroplasty? A metaanalysis of randomized controlled trials? Knee Surg Sports Traumatol Arthrosc. 2012;20:1307-22.

8. Kamat YD, Aurakzai KM, Adhikari AR, Matthews D, Kalairajah Y, Field RE. Does computer navigation in total knee arthroplasty improve patient outcome at midterm followup? Int Orthop. 2009;33:1567-70.

9. Kim CW, Lee CR. Effects of femoral lateral bowing on coronal alignment and component position after total knee arthroplasty: a comparison of conventional and navigationassisted surgery. Knee Surg Relat Res. 2018;30:64-73. 
10. Pfitzner T, Röhner E, Preininger B, Perka C, Matziolis G. Femur positioning in navigated total knee arthroplasty. Orthopedics. 2012;35(10 Suppl):45-9.

11. Nam D, McArthur BA, Cross MB, Pearle AD, Mayman DJ, Haas SB. Patient-specific instrumentation in total knee arthroplasty: a review. J Knee Surg. 2012;25:213-9.

12. Cenni F, Timoncini A, Ensini A, Tamarri S, Belvedere C, D’Angeli V, Giannini S, Leardini A. Three-dimensional implant position and orientation after total knee replacement performed with patient-specific instrumentation systems. J Orthop Res. 2014;32:331-7.

13. Ensini A, Timoncini A, Cenni F, Belvedere C, Fusai F, Leardini A, Giannini S. Intra- and post-operative accuracy assessments of two different patient-specific instrumentation systems for total knee replacement. Knee Surg Sports Traumatol Arthrosc. 2014;22:621-9.

14. Krackow KA, Bayers-Thering M, Phillips MJ, BayersThering M, Mihalko WM. A new technique for determining proper mechanical axis alignment during total knee arthroplasty: progress toward computer-assisted TKA. Orthopedics. 1999;22:698-702.

15. Lopomo N, Sun L, Zaffagnini S, Giordano G, Safran MR.
Evaluation of formal methods in hip joint center assessment: an in vitro analysis. Clin Biomech (Bristol, Avon). 2010;25: 206-12.

16. Brin YS, Livshetz I, Antoniou J, Greenberg-Dotan S, Zukor DJ. Precise landmarking in computer assisted total knee arthroplasty is critical to final alignment. J Orthop Res. 2010; 28:1355-9.

17. Siston RA, Patel JJ, Goodman SB, Delp SL, Giori NJ. The variability of femoral rotational alignment in total knee arthroplasty. J Bone Joint Surg Am. 2005;87:2276-80.

18. Zhang XL, Zhang W, Shao JJ. Rotational alignment in total knee arthroplasty: nonimage-based navigation system versus conventional technique. Chin Med J (Engl). 2012;125:23643.

19. Griffin FM, Math K, Scuderi GR, Insall JN, Poilvache PL. Anatomy of the epicondyles of the distal femur: MRI analysis of normal knees. J Arthroplasty. 2000;15:354-9.

20. Chan WP, Lang P, Stevens MP, Sack K, Majumdar S, Stoller DW, Basch C, Genant HK. Osteoarthritis of the knee: comparison of radiography, CT, and MR imaging to assess extent and severity. AJR Am J Roentgenol. 1991;157:799-806. 